\title{
ACTIVIDAD ELECTROQUÍMICA DE LA CEPA BACTERIANA CrLIM26 ACOPLADA A LA REDUCCIÓN DE Cr(VI)
}

Electrochemical activity of the bacterial strain CrLIM26 coupled to the reduction of $\mathrm{Cr}$ (VI)

Ruby Stella LUCUMÍ BANGUERO ${ }^{1 *}$, Alexander MORA COLLAZOS ${ }^{1}$,
William Hernando LIZCANO-VALBUENA ${ }^{2}$ y Neyla BENÍTEZ-CAMPO

${ }^{1}$ Departamento de Biología, Universidad del Valle, calle 13 No. 100-00, Ciudad Universitaria Meléndez, 76001, Santiago de Cali, Valle del Cauca, Colombia

${ }^{2}$ Departamento de Química, Universidad del Valle, calle 13 No. 100-00, Ciudad Universitaria Meléndez, 76001, Santiago de Cali, Valle del Cauca, Colombia

*Autor para correspondencia: ruby.lucumi@correounivalle.edu.co

(Recibido: septiembre 2018; aceptado: noviembre 2019)

Palabras clave: biorreducción de cromo, densidad de potencia, Exiguobacterium acetylicum, remoción de cromo

\section{RESUMEN}

Las celdas de combustible microbianas (CCM) son una tecnología emergente que aprovecha la degradación de compuestos orgánicos y diferentes sustratos para generar energía, entre los cuales se encuentra el $\mathrm{Cr}(\mathrm{VI})$, metal pesado clasificado como tóxico, mutagénico y cancerígeno para los organismos vivos. El propósito de este trabajo fue determinar la actividad electrogénica acoplada a la biorremediación de $\mathrm{Cr}(\mathrm{VI})$ con la cepa bacteriana CrLIM26, para lo cual se realizaron ensayos de tolerancia al Cr(VI), por concentración mínima inhibitoria, ensayos de reducción del $\mathrm{Cr}(\mathrm{VI})$, identificación filogenética de la cepa bacteriana y se determinó su capacidad de producir energía durante la remoción del Cr(VI) en CCM. La cepa se identificó como Exiguobacterium acetylicum, bacilo corto, Gram positivo, con una tolerancia de $160 \mathrm{mg}$ de $\mathrm{Cr}(\mathrm{VI}) / \mathrm{L}$, con remoción del $100 \%$ del metal en 11 h, a una concentración de $10 \mathrm{mg}$ de $\mathrm{Cr}(\mathrm{VI}) / \mathrm{L}$; presentó actividad electrogénica al producir una densidad de potencia de $107.34 \mathrm{y}$ $89.74 \mu \mathrm{W} / \mathrm{m}^{2}$ en medio LB con y $\sin \mathrm{Cr}(\mathrm{VI})$ respectivamente. Lo anterior permite concluir que la cepa CrLIM26 de E. acetylicum puede ser utilizada para el tratamiento de ambientes contaminados con $\mathrm{Cr}(\mathrm{VI})$ en sistemas acoplados a la generación de energías alternativas en CCM simples.

Key words: chromium bioreduction, chromium removal, Exiguobacterium acetylicum, power density

\begin{abstract}
The microbial fuel cells (MFC) are an emerging technology that takes advantage from organic compounds degradation and different substrates to generate energy. Among these substrates is $\mathrm{Cr}(\mathrm{VI})$, a heavy metal classified as toxic, mutagen and carcinogenic to living organisms. Our purpose was to determine the bioelectrochemical activity, associated with bioremediation of Cr(VI) with the bacterial strain CrLIM26, for which $\mathrm{Cr}$ (VI) tolerance tests were carried out, by minimum inhibitory concentration, reduction assays for $\mathrm{Cr}(\mathrm{VI})$, phylogeny's identification of the bacterial strain and determination
\end{abstract}


of its capacity to produce energy during the removal of $\mathrm{Cr}(\mathrm{VI})$ in MFC. The strain was identified as Exiguobacterium acetylicum, short bacillus, Gram positive, with a $160 \mathrm{mg}$ of $\mathrm{Cr}(\mathrm{VI}) / \mathrm{L}$ tolerance, $100 \%$ metal removal in $11 \mathrm{~h}$, at a concentration of $10 \mathrm{mg}$ of $\mathrm{Cr}(\mathrm{VI}) / \mathrm{L}$, with bioelectrochemical activity producing a power density of 107.34 and $89.74 \mu \mathrm{W} / \mathrm{m}^{2}$ in LB medium with and without $\mathrm{Cr}(\mathrm{VI})$ respectively. The above allows to conclude that the strain CrLIM26 of E. acetylicum can be used for treatment contaminated environments with $\mathrm{Cr}(\mathrm{VI})$ in systems associated to the generation of alternative energies in MFCs.

\section{INTRODUCCIÓN}

El cromo presenta cinco estados de valencia, de los cuales los más importantes y estables en medios naturales son el cromo (III) y (VI), que se encuentran presentes en acuíferos naturales y aguas residuales (Cheung y Ji 2007, Dhal et al. 2013, Evelyne y Ravisanka 2014, Tommasi y Lombardelli 2017). El Cr(VI) es considerado un agente mutagénico, carcinogénico y teratogénico, el cual se presenta como cromato o dicromato, dependiendo del $\mathrm{pH}$ en donde se encuentre (Dhal et al. 2013, Suh et al. 2019). Debido a su naturaleza química, no puede degradarse, sólo transformarse a una forma menos tóxica como es el $\mathrm{Cr}$ (III), el cual es esencial para el metabolismo humano en bajas concentraciones, ya que participa en el metabolismo de la glucosa, el colesterol y los ácidos grasos, donde desempeña un papel importante en diferentes reacciones enzimáticas (Arderiu 1998, Watson y Logan 2011, Dhal et al. 2013, Suh et al. 2019). El Cr(III) considerado 100 veces menos tóxico y 1000 veces menos mutagénico que el Cr(VI) (Dhal et al. 2013, Kanmani et al. 2012, Guan et al. 2019, Suh et al. 2019) se encuentra en la naturaleza formando hidróxidos, óxidos o sulfatos, los cuales son solubles en agua.

Para el tratamiento de la contaminación por Cr(VI) existen diferentes estrategias como el uso resinas de intercambio iónico, los procesos de filtración y los métodos de reducción química. Sin embargo, estas estrategias no son limpias, usualmente tienen altos costos y generan productos secundarios, que a su vez originan otros problemas de contaminación. Por lo tanto, se plantea el uso de alternativas que sean amigables con el ambiente, como es el caso de la biorremediación (Rehman et al. 2008, Cárdenas et al. 2011). Método que se basa en el aprovechamiento de las características de diferentes organismos como plantas, levaduras, hongos y bacterias, entre otros (Alcalá 2007, Gaun et al. 2019), los cuales poseen diferentes mecanismos para retener, degradar o transformar sustancias tóxicas, siendo una de ellas el Cr(VI) (Rehman et al. 2008, Benítez-Campo 2011, Thatoi et al. 2014).
Adicionalmente, la biorremediación puede combinarse con la tecnología de las celdas de combustible microbianas (CCM) en la cual se emplean aguas residuales domésticas o industriales como sustrato directo para producir espontáneamente energía, a la vez que se disminuye la concentración de diferentes contaminantes (Min y Logan 2004, Thygesen et al. 2011, Wang et al. 2008, Zhang et al. 2016, Liu et al. 2019).

En las CCM la energía química contenida en la materia orgánica se transforma por los microorganismos en energía eléctrica en el ánodo. El circuito se cierra con un medio electrolítico, un circuito externo (Bond et al. 2002) y un cátodo recubierto generalmente con platino (Li et al. 2009, Revelo et al. 2018). Un aspecto importante de las CCM es el tipo de sustrato que se emplea, ya que a partir de este se produce la energía. En la literatura se reportan desde compuestos puros hasta mezclas complejas, como es el caso de sedimentos de aguas marinas (Reimers 2001, Logan et al. 2006), agua contaminada con $\mathrm{Cr}(\mathrm{VI})$, aguas residuales domésticas (Sophia y Sai 2016a, 2016b), celulosa, acetato, glucosa y lactosa entre otras sustancias (Pant et al. 2010, Zhang et al. 2016, Kim et al. 2017, Loloei et al. 2017, Tommasi y Lombardelli 2017).

Además, los microorganismos que se emplean se caracterizan por ser anaerobios facultativos o anaerobios estrictos, no fermentativos y electroquímicamente activos (exoelectrogénicos), entre los cuales se han reportado bacterias como Shewanella oneidensis, Geobacter sulforreducens, Geobacter metallireducens, Rhodoferax ferrireducens, Klebsiella pneumoniae, Clostridium butyricum, y Exiguobacterim acetylicum (Debabov 2008, Wang et al. 2008, Xing et al. 2008, Vejarano et al. 2018).

En estudios previos realizados por Mora y Bravo (2017), se aisló la cepa CrLIM26 del electrodo de una CCM ubicada en el lago de la estación experimental del Departamento de Biología de la Universidad del Valle. Esta cepa presentó actividad electroquímica y, considerando dicho antecedente, el objetivo de este trabajo fue determinar la actividad electrogénica acoplada a la biorremediación de cromo hexavalente con la cepa 
bacteriana CrLIM26. Para esto se realizaron ensayos de tolerancia al $\mathrm{Cr}(\mathrm{VI})$ por concentración mínima inhibitoria (CMI) y ensayos de reducción del Cr(VI). Asimismo, se hizo la identificación filogenética de la cepa bacteriana y se evaluó su capacidad para producir energía durante la remoción del $\mathrm{Cr}(\mathrm{VI})$ en CCM.

\section{MATERIALES Y MÉTODOS}

Este trabajo fue realizado en el Laboratorio de Investigaciones Microbiológicas (LIM) del Departamento de Biología de la Universidad del Valle, Santiago de Cali, Colombia, $3^{\circ} 22^{\prime} 30^{\prime \prime} \mathrm{N}$ $76^{\circ} 32^{\prime} 04^{\prime \prime} \mathrm{O}$.

\section{Ensayo de tolerancia al Cr(VI)}

Para determinar la tolerancia de la cepa CrLIM26 al Cr(VI), se utilizó el método de concentración mínima inhibitoria (CMI) en el medio de cultivo $\mathrm{LB}$, inoculado con $10 \% \mathrm{~V} / \mathrm{V}$ de un cultivo fresco de la cepa CrLIM26 y suplementado con Cr(VI) a concentraciones de 5, 10, 20, 40, 80, 160, 320, 640, 1280 y $2560 \mathrm{mg} / \mathrm{L}$, ajustado a un volumen final de $2 \mathrm{~mL}$. Se usó un testigo positivo sin $\mathrm{Cr}(\mathrm{VI})$ y un testigo negativo sin inóculo, con el fin de garantizar la inocuidad de los tratamientos y verificar si existía reducción química espontánea. Todas las pruebas se realizaron por triplicado. La CMI fue interpretada como la concentración de $\mathrm{Cr}(\mathrm{VI})$ a la cual se inhibió el crecimiento visible de la cepa después de $24 \mathrm{~h}$ de incubación por comparación con el crecimiento del testigo positivo y las diferentes soluciones evaluadas (Rangdale et al. 1997, Andrews 2001). El crecimiento se midió por espectrofotometría a una longitud de onda de $550 \mathrm{~nm}$.

Estimación del potencial de reducción del Cr(VI)

En pruebas preliminares se determinó que la cepa CrLIM26 presentó condiciones óptimas de crecimiento a $35^{\circ} \mathrm{C}$ y pH $7.0(\mathrm{p}=0.00321$ ensayos no mostrados en el presente trabajo), motivo por el cual los bioensayos de reducción de cromo se realizaron a esas condiciones de incubación. Todos los bioensayos de remoción de $\mathrm{Cr}(\mathrm{VI})$ se realizaron por triplicado, en matraces Erlenmeyer a los cuales se les agregaron $45 \mathrm{~mL}$ de caldo LB, $100 \mu \mathrm{L}$ de una solución patrón de $\mathrm{Cr}(\mathrm{VI})$, para garantizar una concentración final de $10 \mathrm{mg} / \mathrm{L}$ de $\mathrm{Cr}$ (VI) y $5 \mathrm{~mL}$ del inoculo bacteriano de 14-16 h de incubación. Se dispuso un testigo positivo de crecimiento sin cromo y un testigo negativo con cromo, pero sin inoculo bacteriano. Las variables de respuesta fueron la concentración de $\mathrm{Cr}(\mathrm{VI})$ y el crecimiento bacteriano; la concentración de cromo se estimó por el método de la 1.5 difenilcarbazida usando un espectrofotómetro Hach DR/890 (Ramírez y Benítez 2013) y el crecimiento bacteriano se midió por densidad óptica a $550 \mathrm{~nm}$. Dichas mediciones se realizaron cada $2 \mathrm{~h}$, hasta que la concentración de cromo fue imperceptible.

\section{Efecto del Cr(VI) en la morfología de la cepa CrLIM26}

El efecto del Cr(VI) sobre la morfología bacteriana se observó por microscopía electrónica de barrido. Para esto se realizaron dos cultivos de la cepa CrLIM26 en caldo LB con y sin Cr(VI), a los cuales se les agregó una barra de grafito como soporte de fijación para las bacterias, dejándose en incubación a temperatura ambiente durante 15 días. Para el proceso de fijación se tomaron las barras de grafito y se sumergieron en solución de glutaraldehído a $2.5 \%$ en amortiguador de fosfato de sodio $0.1 \mathrm{M}$ a pH 7.2 por $42 \mathrm{~h} \mathrm{a} 4{ }^{\circ} \mathrm{C}$. Después de retirar el glutaraldehído se les agregó solución fría de etanol a $10 \%, 30 \%, 40 \%, 50 \%$, $60 \%, 70 \%, 80 \%, 90 \%$ y $100 \%$, cada solución se incubó a $4{ }^{\circ} \mathrm{C}$ durante $10 \mathrm{~min}$. Las muestras se retiraron del etanol e inmediatamente fueron secadas agregando gotas de hexametildisiloxano (HMDS). Las muestras se adhirieron a un soporte metálico y se mantuvieron en desecador con sílica gel hasta su recubrimiento con oro en el equipo Sputter Coater - Denton Vacuum Desk IV para la observación en microscopio electrónico de barrido (Joel JSM 6490LV).

\section{Identificación de la cepa CrLIM26}

Para la identificación de la cepa CrLIM26, se realizó la extracción del ADN genómico utilizando el paquete de extracción Ultraclean Microbial DNA isolation de Mobio, siguiendo las indicaciones del fabricante. A partir del ADN obtenido se amplificó por PCR el gen 16S rRNA utilizando los cebadores universales 27F (5'-AGAGTTTGATCMTGGCTCAG3') y 1492R (5'TACGGYTACCTTGTTACGACTTT'3). La mezcla de la PCR se preparó con base en las siguientes concentraciones finales: solución amortiguadora (1X) $\mathrm{MgCl}_{2}(1.75 \mu \mathrm{M})$ DNTPs $(20 \mu \mathrm{M}$ cada uno $)$ cebadores ( $4 \mu \mathrm{M}$ cada uno) polimerasa Taq ( 1 unidad). El programa utilizado para la amplificación consistió en un ciclo de desnaturalización a $94{ }^{\circ} \mathrm{C}$ durante 5 min, una fase de amplificación de 35 ciclos con una desnaturalización a $94{ }^{\circ} \mathrm{C}$ durante $1 \mathrm{~min}$, hibridación a $54{ }^{\circ} \mathrm{C}$ durante $45 \mathrm{~s}$ y extensión a $72{ }^{\circ} \mathrm{C}$ durante $40 \mathrm{~s}$; seguido de una extensión final a $72^{\circ} \mathrm{C}$ durante $10 \mathrm{~min}$. Se determinaron las secuencias mediante procedimientos estándares del servicio suministrado por la 
compañía Macrogen (EUA), amparados por la Resolución 1070 (MADS, ANLA 2015).

Las secuencias obtenidas fueron comparadas directamente con la base de datos del Genbank y del Ribosomal Database Project, para clasificar y asignar los taxones jerárquicos. Se colectaron secuencias de las especies registradas para el género Exiguobacterium y fueron alineadas con el programa Bioedit Sequence Alignment para la construcción de los árboles filogenéticos mediante el método de máxima verosimilitud utilizando el programa PAUP versión $4.0 \mathrm{~b} 10$ con el algoritmo de TBR (Tree-BisectionReconnection). El modelo de sustitución nucleotídica fue seleccionado por el criterio de Akaike en Modeltest versión 2.1; el soporte estadístico fue evaluado con el método "bootstrap" de máxima parsimonia con 10000 repeticiones (MP), máxima verosimilitud 1000 repeticiones (ML) y análisis de inferencia bayesiana con 1000000 de remuestreos, tomando muestras cada 100 generaciones. Se generó un árbol consenso con el $50 \%$ de los árboles más representativos. Esta identificación se complementó con la realización de pruebas bioquímicas, resultados no mostrados en este trabajo.

\section{Monitoreo de actividad electroquímica en presen- cia y ausencia de $\mathrm{Cr}$ (VI)}

Se construyeron cuatro celdas de combustible microbianas (CCM) de una sola cámara (simples), fabricadas en vidrio con un volumen de $100 \mathrm{~mL}$. En cada una de ellas se colocaron dos colectores de corriente anódica y catódica, compuestos por grafito con dimensiones de $8 \mathrm{~mm}$ de diámetro y $5 \mathrm{~cm}$ de longitud, con área superficial de $13.57 \mathrm{~cm}^{2}$. Con la ayuda de un taladro se realizaron orificios para introducir un alambre de cobre, que se conectó al circuito. El colector catódico fue recubierto con $\mathrm{Pt} / \mathrm{C}$ hasta depositar $0.416 \mathrm{mg} / \mathrm{cm}^{2}$ de platino a partir de una mezcla de $\mathrm{Pt} / \mathrm{C}$ $20 \%$ de peso de $\mathrm{Pt} /$ (peso de $\mathrm{Pt}+$ peso del soporte de carbono), $50 \mu \mathrm{L}$ de Nafion en solución al $5 \%$ en alcoholes de bajo peso molecular y $50 \mu \mathrm{L}$ de isopropanol. $\mathrm{La}$ mezcla fue homogeneizada en un ultrasonido por $1 \mathrm{~h}$, y luego se aplicaron tres capas de la solución a la barra de grafito, dejando secar cada capa a temperatura ambiente, con el fin de obtener una capa homogénea.

Se usaron tres celdas iguales, con un volumen de $67.5 \mathrm{~mL}$ de medio LB, más $7.5 \mathrm{~mL}$ de inóculo de la cepa CrLIM26; el ánodo se ubicó en la parte inferior para mantenerlo en condiciones anaerobias y el cátodo en la parte superior en contacto con el aire, la separación entre los electrodos fue de $2 \mathrm{~cm}$. La cuarta celda con un volumen de trabajo de $75 \mathrm{~mL}$ de caldo LB, se empleó como testigo.
Se obtuvo una curva de polarización de acuerdo con Xing et al. (2008), al medir el voltaje (V) y la corriente (I) usando resistencias externas $\left(\mathrm{R}_{\mathrm{ex}}=1 \Omega\right.$ a $3900 \mathrm{k} \Omega$ ), conectadas a un circuito eléctrico con dos multímetros. Para la toma de datos se usó una fase de estabilización de $15 \mathrm{~min}$, después con cada resistencia se realizaron tres ciclos de 5 min y se tomaron los datos de voltaje (V) y la corriente (I) generados en el último ciclo. La potencia $(\mathrm{P})$ se calculó a partir de $\mathrm{P}=\mathrm{IV}$, la densidad de potencia (DP) fue igual a $\mathrm{P} / \mathrm{A}_{\text {cat }}$, donde $\mathrm{A}_{\text {cat }}$ es el área del cátodo usado, y la densidad de corriente (DI) fue igual a I/ $\mathrm{A}_{\text {cat }}$, los datos fueron normalizados tomando como referencia el área del cátodo (Martinez-Santacruz et al. 2016). La curva de densidad de corriente se construyó usando los valores de DI máxima generada diariamente. Adicionalmente, para evaluar la cantidad de energía que se produce al reducir $\mathrm{Cr}(\mathrm{VI})$, se agregaron 150 $\mu \mathrm{L}$ de la solución patrón de $\mathrm{Cr}(\mathrm{VI})$, para obtener una concentración final de $10 \mathrm{mg} / \mathrm{L}$ de $\mathrm{Cr}(\mathrm{VI})$, a cada celda. Inmediatamente se inició el monitoreo de la concentración de $\mathrm{Cr}(\mathrm{VI})$ con mediciones cada hora hasta su agotamiento. La adición del metal se hizo los días 14 y 28 después de iniciado el experimento, el voltaje se midió usando una resistencia de $1 \mathrm{k} \Omega$.

\section{RESULTADOS}

\section{Estimación del potencial de reducción de $\mathrm{Cr}(\mathrm{VI})$}

Los resultados de los bioensayos mostraron que el crecimiento de la cepa siguió una cinética de orden 1 , con una disminución leve cuando se comparó con el testigo positivo (ausencia de $\mathrm{Cr}(\mathrm{VI})$ ). El microorganismo redujo durante las primeras $6 \mathrm{~h}$ de crecimiento el $60 \%$ de la concentración del cromo, alcanzando la remoción del $100 \%$ de la concentración del metal en $11 \mathrm{~h}$ (Fig. 1), mientras que el testigo negativo reveló que no hubo reducción abiótica en el medio LB.

\section{Efecto del $\mathrm{Cr}(\mathrm{VI})$ en la morfología de la cepa $\mathrm{Cr}$ - LIM26 y concentración mínima inhibitoria (CMI)}

La cepa CrLIM26 en ausencia de Cr(VI) presenta forma de bacilos cortos de 1 a $2 \mu \mathrm{m}$ de longitud, móviles, Gram positivos, sin endosporas, agrupados en pares o en forma individual (Fig. 2a). Las colonias en agar LB, prestaron forma redondeada, de color crema, con borde entero y elevadas, se tornaron de color naranja después de 24 h. Esta coloración no se presentó cuando el microorganismo fue cultivado en anaerobiosis. Mientras que en presencia de $\mathrm{Cr}(\mathrm{VI})$ las células de CrLIM26 se tornaron más largas y anchas, formando agregados (Fig. 2b). Además de 


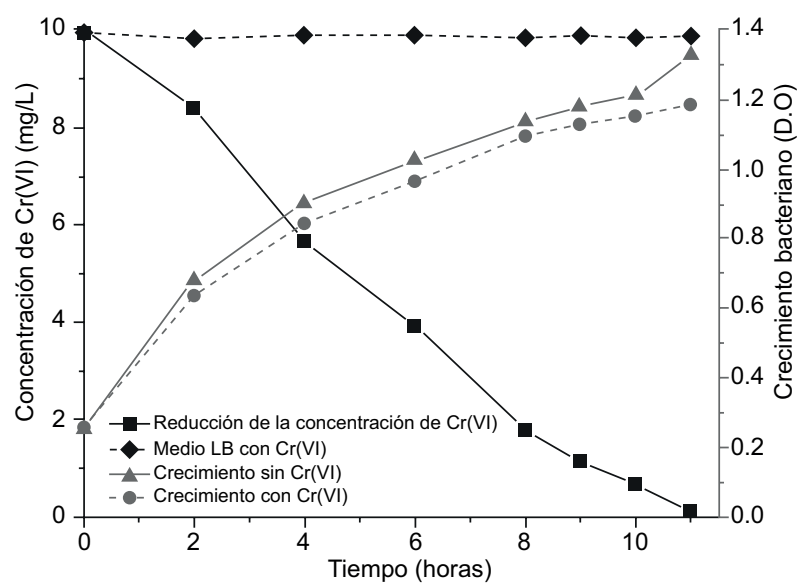

Fig. 1. Cinética de crecimiento y reducción de la concentración de $\mathrm{Cr}(\mathrm{VI})$ en horas (h) de la cepa CrLIM26, mediante densidad óptica (D.O.)

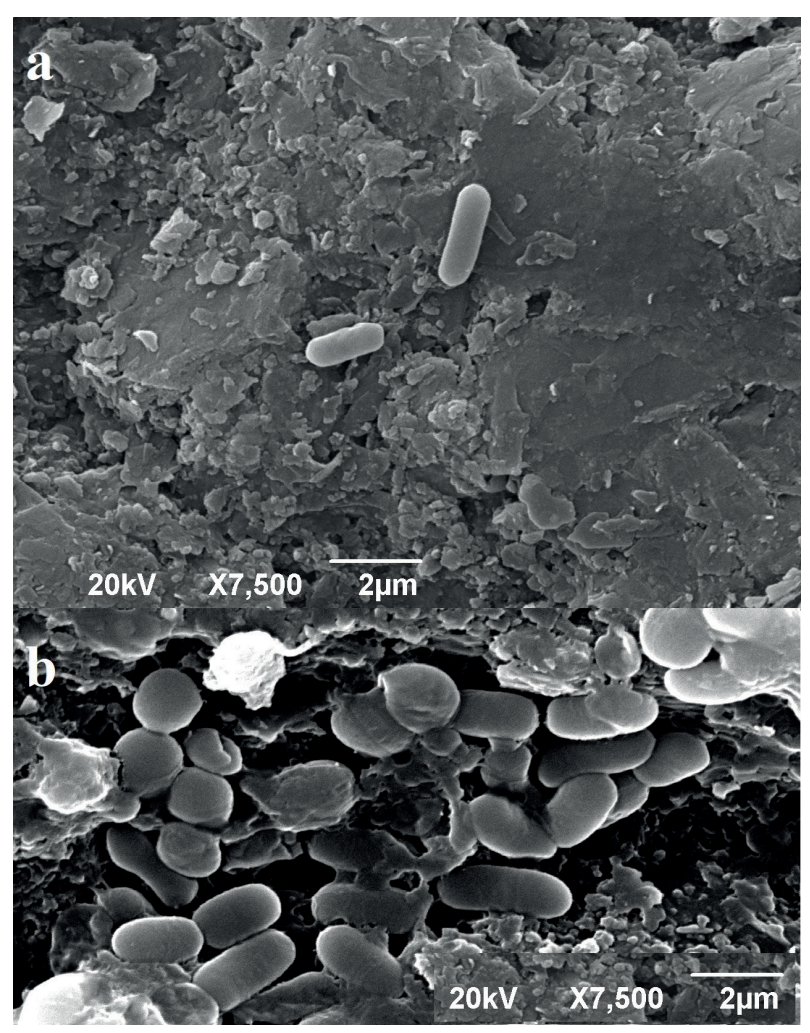

Fig. 2. Microscopia electrónica de barrido de células de la cepa CrLIM26 incubadas en ausencia (a) y en presencia de $\mathrm{Cr}(\mathrm{VI})$ (b). Aumento $7500 \mathrm{X}$

los cambios morfológicos se evidenció la reducción del crecimiento bacteriano de la cepa en estudio, ocasionada por la toxicidad del cromo, pues el crecimiento se afectó a concentraciones superiores a $160 \mathrm{mg} / \mathrm{L}$ de $\mathrm{Cr}(\mathrm{VI})$.

\section{Identificación de la cepa CrLIM26}

La cepa CrLIM26 se identificó como Exiguobacterium acetylicum, según se indica en el árbol filogenético obtenido por el método de máxima verosimilitud (Fig. 3) con el modelo de sustitución $\mathrm{GRT}+\mathrm{I}+\mathrm{G}$, donde la cepa se agrupó en el clado conformado por Exiguobacterium indicum y Exiguobacterium acetylicum.

\section{Monitoreo de actividad electroquímica en presen- cia y ausencia de $\mathrm{Cr}(\mathrm{VI})$}

En las CCM operando con medio LB se tomaron los valores de voltaje y corriente durante 30 días, observándose diariamente valores para estos parámetros similares en las tres réplicas. En la figura 4a se observa la curva de polarización para el día de mayor densidad de potencia (día 25), con una resistencia externa de $3 \mathrm{k} \Omega$ alcanzando un máximo de $89.74 \mu \mathrm{W} / \mathrm{m}^{2}\left(\mathrm{DI}=4.75 \mathrm{~mA} / \mathrm{m}^{2}\right.$ y V=18.88 mV).

En las CCM, al adicionar el Cr(VI) durante las primeras dos horas, se observó la disminución de la densidad de potencia (Fig. 4b), pero una vez la concentración del cromo (VI) alcanzó $50 \%$ de la adicionada, los valores de la densidad de potencia comenzaron a incrementarse. Nótese que la reducción de $100 \%$ de la concentración de $\mathrm{Cr}(\mathrm{VI})$ en la CCM, ocurrió después de $6 \mathrm{~h}$, siendo mucho más rápido que en los bioensayos de reducción de cromo iniciales. Igualmente se alcanzó una DP final de $107.34 \mu \mathrm{W} / \mathrm{m}^{2}$, mayor a la inicial que fue de $96.1 \mu \mathrm{W} / \mathrm{m}^{2}$; esta tendencia se observó en los dos días evaluados, el día 14 y el día 28 .

\section{DISCUSIÓN}

La reducción del $60 \%$ del Cr(VI) por el aislado bacteriano CrLIM26 se produjo durante las primeras 6 horas de crecimiento, coincidiendo con la fase exponencial, mientras que el $40 \%$ restante se removió más lentamente durante la fase estacionaria. Este fenómeno está asociado al crecimiento bacteriano, lo cual se evidencia en estudios similares, en los que se demostró que durante la fase exponencial también existe un aumento en la velocidad de reducción del $\mathrm{Cr}(\mathrm{VI})$ ligada a los procesos metabólicos que generan un crecimiento en la población bacteriana (Ramírez y Benítez 2013, Guerrero et al. 2017). Es importante señalar que el testigo negativo no presentó reducción de $\mathrm{Cr}(\mathrm{VI})$, evidenciando que ningún componente del medio utilizado fue un agente reductor de $\mathrm{Cr}(\mathrm{VI})$.

El crecimiento celular de la cepa CrLIM26 disminuyó levemente en comparación con el testigo 


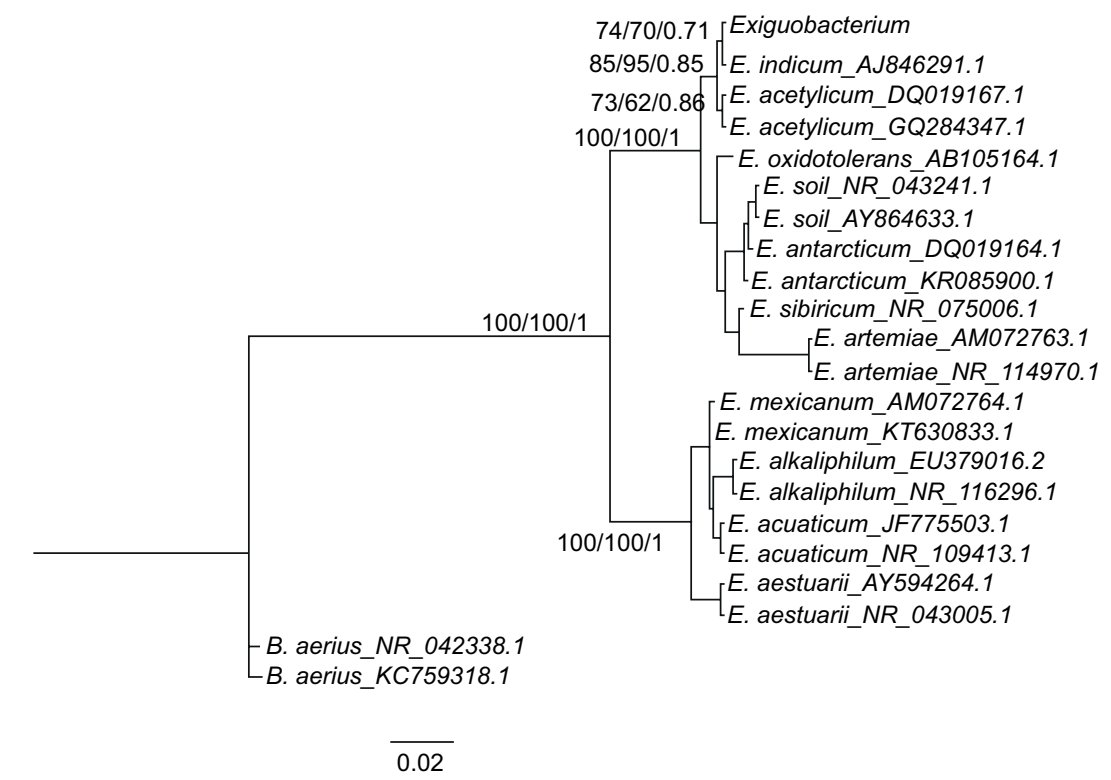

Fig. 3. Árbol filogenético de la cepa CrLIM26 entre organismos del género Exiguobacterium, basados en las secuencias del gen ARNr 16S, con el grupo externo de Bacillus aerius, los valores en las ramas indican máxima parsimonia, máxima verosimilitud y valor de inferencia bayesiana (izquierda a derecha)
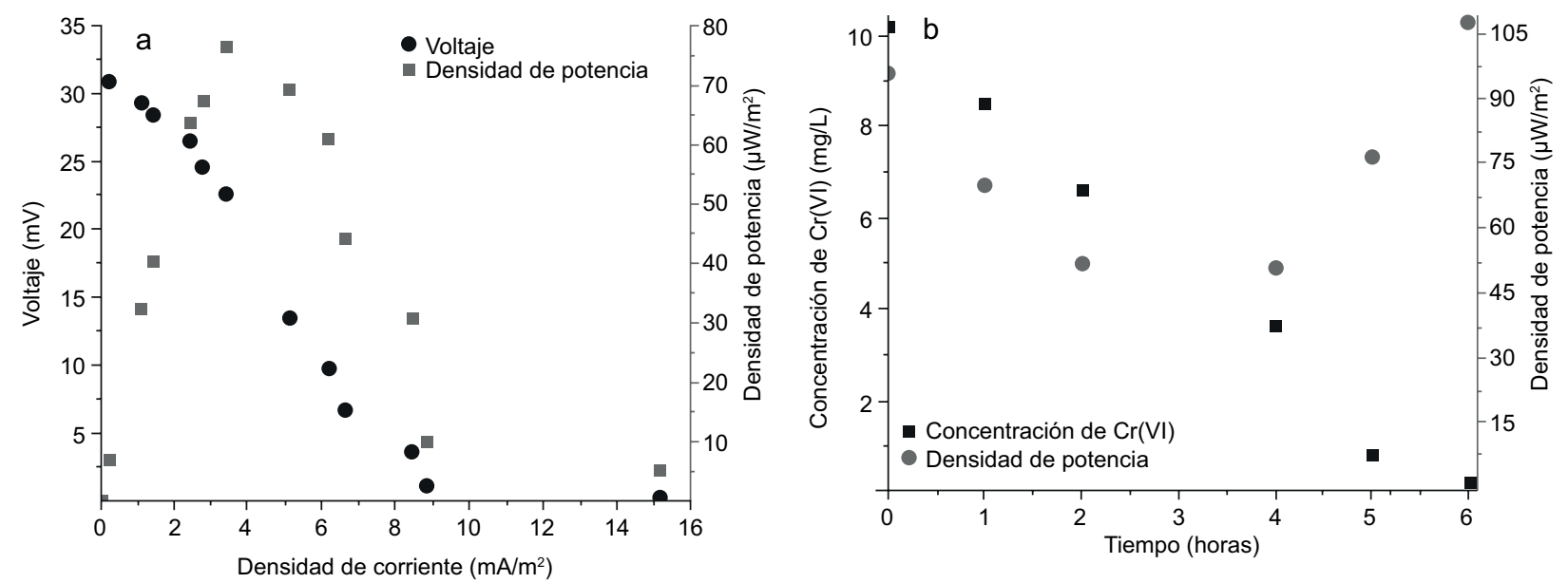

Fig. 4. Curva de polarización y de densidad de corriente máxima obtenida para la cepa CrLIM26 en las celdas de combustible microbianas $(\mathrm{CCM})$ en ausencia de $\mathrm{Cr}(\mathrm{VI})$ (a), reducción de la concentración de $\mathrm{Cr}(\mathrm{VI})$ y densidad de corriente generadas en las CCM en presencia de cromo (b) medido a través del tiempo en horas (h)

positivo que no contenía $\mathrm{Cr}(\mathrm{VI})$, este resultado puede deberse a que, bajo condiciones de estrés inducido por el $\mathrm{Cr}(\mathrm{VI})$, las bacterias activan mecanismos de absorción o expulsión de iones o cascadas redox asociadas a rutas metabólicas. Esto genera una inversión energética en procesos de transporte activo para controlar la entrada de este metal altamente toxico, generando una disminución en el proceso de división celular y provocando que sea más lento el crecimiento celular (Morais et al. 2011, Thatoi et al. 2014, Viti et al. 2014, Guerrero et al. 2017).

La cepa CrLIM26 sufrió cambios morfológicos en presencia de $\mathrm{Cr}(\mathrm{VI})$ formando agregados y su morfología celular se tornó más larga y ancha, en comparación con la morfología en ausencia de Cr(VI), (Fig. 2). Los cambios morfológicos en presencia de $\mathrm{Cr}(\mathrm{VI})$ como son la deformación y el aumento de tamaño celular en bacterias se atribuye 
a la acumulación de este metal en el interior celular, según Sarangi y Krishnan (2008), Recillas et al. (2010), Morais et al. (2011), Singh et al. (2015) y Echeverry (2016).

La acumulación del $\mathrm{Cr}(\mathrm{VI})$ en el interior celular ha sido asociada a la presencia de grupos funcionales amino, carboxilos, carbonilo e hidroxilos en la superficie de la membrana celular, que facilita el ingreso de este metal al interior celular (Oves et al. 2013, Guerrero et al. 2017). Adicionalmente, la formación de agregados o biopelículas, proporciona una cooperación fisiológica en la células y protección contra diferentes condiciones ambientales hostiles o de estrés ambiental, brindando mayor resistencia y tolerancia a agentes peligrosos como el Cr(VI) (Loera et al. 2012, Olarte y Cristiano 2012, Mendoza et al. 2015).

La cepa CrLIM26 presentó una CMI de $160 \mathrm{mg}$ $\mathrm{Cr}(\mathrm{VI}) / \mathrm{L}$, resultado asociado a la acumulación en el interior celular y a los diferentes mecanismos de resistencia que se han reportado para microorganismos similares, los cuales son variados y pueden ser conferidos por genes localizados en los cromosomas o en plásmidos (Morais et al. 2011). Por lo general, estos mecanismos son codificados por genes que permiten la expulsión del cromato a través de la membrana. Otros sistemas de resistencia están relacionados con estrategias como la reducción del $\mathrm{Cr}(\mathrm{VI})$ de forma enzimática específica o no específica, o por actividad de enzimas de destoxificación de especies reactivas de oxígeno (ROS). Finalmente, estos procesos pueden estar asociados con la homeostasis de azufre, hierro, sulfatos y/o por reparación de daños del ADN (Morais et al. 2011, Thatoi et al. 2014, Viti et al. 2014).

La cepa CrLIM26 se identificó como Exiguobacterium acetylicum, resultado que se confirmó con las pruebas bioquímicas realizadas. Otros autores como Okeke (2008), Sarangi y Krishnan (2008) y Zumbair y Malik (2008), han reportado la capacidad de esta especie para reducir $\mathrm{Cr}(\mathrm{VI})$ a concentraciones mayores o menores a la presentada en este estudio. Sin embargo, no se ha informado el modo de reducción del Cr(VI) en este género.

En cuanto a la evaluación electroquímica de la cepa $E$. acetylicum CrLIM26, esta generó una densidad de potencia máxima de $89.74 \mu \mathrm{W} / \mathrm{m}^{2}$ a una densidad de corriente de $4.75 \mathrm{~mA} / \mathrm{m}^{2}$. Esta especie, en estudios preliminares mostró una densidad de potencia de $6.9 \mathrm{~mW} / \mathrm{m}^{2}$ (Mora y Bravo 2017). Estos resultados contrastan con la potencia generada al usar distintos microorganismos como Desulfovibrio desulfuricans (Pant et al. 2010), Geobacter sulfurreducens (Nevin et al. 200), Rhodopseudomonas palustris (Xing et al, 2008) y Geobacter sulfurreducens (Bond y Lovley 2003), los cuales generaron potencias máximas de $1150 \mathrm{~mA} / \mathrm{m}^{2}, 2.15 \mathrm{~kW} / \mathrm{m}^{3}, 1170 \mathrm{~mW} / \mathrm{m}^{2}$ y $1150 \mathrm{~mA} / \mathrm{m}^{2}$, respectivamente. Esto demuestra la relación entre la bacteria usada, la corriente y el voltaje generado, causado por la diferencia en la actividad metabólica, el mecanismo de transferencia de electrones al colector de corriente por parte de cada bacteria y los factores asociados al sistema como el diseño, la resistencia usada en el circuito, el suministro de oxígeno y el tiempo de funcionamiento de las celdas (Min y Logan 2004, He et al. 2005, Debabov 2008, Watson y Logan 2011, Chen et al. 2014, Parkash 2016, Penteado et al. 2016)

La curva de polarización (Fig. 4a) mostró un aumento paulatino del desempeño electroquímico, lo que indica un crecimiento en la colonización de la superficie del ánodo (Mendoza et al. 2015, Revelo et al. 2018), incrementando el número de bacterias por área y generando un mayor flujo de electrones que entran al circuito externo de las celdas (Vejarano et al. 2018, Revelo y Hurtado 2013). Adicionalmente, en las curvas de voltaje vs DI se observó que la pendiente fue poco pronunciada, lo que indica una mejora en la capacidad del sistema al dejar pasar mayor cantidad de corriente (Vejarano et al. 2018). En otros trabajos se argumenta que esto se relaciona con la maduración de la biopelícula donde la población bacteriana logra establecerse, multiplicarse y adaptarse al medio (Bond y Derek 2003, Watson y Logan 2011).

La DI alcanzó su valor máximo en los primeros 15 días, tiempo en el cual se presume el establecimiento de una biopelícula bacteriana sobre los ánodos de la CCM. Posterior al establecimiento de la biopelícula, los microorganismos trasmiten electrones de forma óptima desde la matriz celular hasta los electrodos del dispositivo; proceso mediado por contacto directo o mediante nanocables y nanohilos conductivos, que facilitan la transferencia de electrones (Min y Logan 2004, Debabov 2008, Fernández-Marchate et al. 2016, Parkash 2016, Bose et al. 2018, Li et al 2018).

Un factor que influyó durante todo el proceso fue la cantidad de nutrientes disponibles en el medio durante el experimento. Se ha demostrado que a mayor cantidad de materia orgánica, mayor es la cantidad de corriente generada, ya que a partir del sustrato se produce la energía (Min y Logan 2004, Cheng y Logan 2011, Sophia y Sai 2016a, Bose et al. 2018, Vejarano et al. 2018). Otro factor influyente es la actividad microbiana debida a la naturaleza misma de las bacterias, que emplean mecanismos específicos 
de transferencia de carga al colector anódico y tipo de sustrato (Bond et al. 2002, Li et al. 2009, Sophia y Sai 2016b). Adicionalmente, Martínez-Santacruz et al. (2016) consideran que las resistencias bajas favorecen el desarrollo de biopelículas de microorganismos exoelectrogénicos.

Wartson y Logan (2011), encontraron que las biopelículas necesitan un tiempo más largo de adaptación a las resistencias aplicadas, recomendando el uso de resistencias fijas. Ellos reconocen que dichas características pueden producir un incremento de la corriente y la potencia, al igual que el suministro de oxígeno (He et al. 2005, Watson y Logan 2011, Martínez-Santacruz et al. 2016, Parkash 2016). Asimismo, se ha demostrado que el uso de electrodos de grafito, empleados por su relación costo beneficio en lugar de colectores metálicos, puede proporcionar una superficie de adherencia para las bacterias (Logan et al. 2007, Loloei et al. 2017, Li et al 2018).

En cuanto a la producción de energía de la CCM en presencia de $\mathrm{Cr}(\mathrm{VI})$, se encontró un efecto negativo al inicio del experimento en términos de la producción de potencia, asociada al uso del $\mathrm{Cr}(\mathrm{VI})$ como aceptor de electrones en el cátodo. Otros reportes hechos por Wang et al. (2008), Li et al. (2009), Huang et al. (2011), Wu et al. (2015), Carmalin y Saikant (2016) y Kim et al. (2017), sugieren que la disminución en la producción de energía está asociada al poder electronegativo del $\mathrm{Cr}(\mathrm{VI})$ que al agotarse deja de funcionar como un sumidero de electrones y el voltaje vuelve a restablecerse, situación coherente con la evidencia experimental de la presente investigación.

También, se plantea que el Cr(VI) no sólo está siendo reducido por la actividad bacteriana, sino por actividad electroquímica, lo que genera una disminución en la producción de potencia de la CCM y una aceleración del proceso de reducción de $\mathrm{Cr}(\mathrm{VI})$ en el medio (Huang et al. 2011, Wei et al. 2011, Wu et al. 2015, Carmalin y Saikant 2016, Loloei et al. 2017). Considerando que el $\mathrm{Cr}(\mathrm{VI})$ es un agente oxidante, podría ser que la actividad electroquímica encontrada y su efecto en la reducción del Cr(VI) resultara de una combinación de factores, tales como: cantidad de biomasa, crecimiento celular en su fase exponencial y mecanismos de adsorción y reducción del cromo, pues a mayor cantidad de inóculo, hay más sitios de unión al metal (Cárdenas et al. 2011, Wu et al. 2015, Carmalin y Saikant 2016, Loloei et al. 2017, Revelo et al. 2018), además de un aumento en la actividad electroquímica por parte del microorganismo.

\section{CONCLUSIONES}

En este trabajo la cepa nativa E. acetylicum $\mathrm{Cr}-$ LIM26 mostró, a pesar del efecto tóxico del metal, la capacidad de tolerar y disminuir la concentración de $\mathrm{Cr}(\mathrm{VI})$ en caldo LB, evidenciado por un mayor volumen y longitud celular. Se demostró la transferencia de electrones en presencia y ausencia de $\mathrm{Cr}(\mathrm{VI})$ en celdas de combustible microbianas, mostrando su potencial para la biorremediación de ambientes contaminados con este ión en futuros estudios con sistemas acoplados a la generación de energías alternativas en CCM.

En futuros estudios podría evaluarse la eficiencia y factibilidad de la aplicación del sistema de CCM empleando E. acetylicum cepa CrLIM26 como inóculo masivo, para la remoción de $\mathrm{Cr}(\mathrm{VI})$ en residuos industriales o en ambientes contaminados con este metal.

\section{AGRADECIMIENTOS}

A la Universidad del Valle por la financiación del proyecto C.I 7971, al personal del Laboratorio de Investigaciones Microbiológicas por el uso de las instalaciones y la asesoría durante la ejecución del proyecto.

\section{REFERENCIAS}

Alcalá M. (2007). La contaminación de suelos y aguas: su prevención con nuevas sustancias naturales. Universidad de Sevilla. Sevilla, España, 243 pp.

Andrews J.M. (2001). Determination of minimum inhibitory concentrations. J. Antimicrob. Chemoth. 48 (Suppl. 1), 5-16. https://doi.org/10.1093/jac/48. suppl_1.5

Arderiu X. F. (1998). Bioquímica clínica y patología molecular. Vol. 2. Reverté, Barcelona, España, 540 pp.

Benítez-Campo N. (2011). Producción limpia y biorremediación para disminución de la contaminación por cromo en la industria de curtiembres. Ambiente y Sostenibilidad 1 (1), 25-31.

Bond D. y Derek R. (2003). Electricity production by Geobacter sulfurreducens attached to electrodes. Appl Environ. Microb. 69 (3), 1548-55. https://doi. org/10.1128/AEM.69.3.1548-1555.2003

Bond D., Hlmes L., Tende L. y Lovley D. (2002). Electrode-reducing microorganisms that harvest energy from marine sediments. Science 295 (5554), 483-85. https://doi.org/10.1126/science.1066771 
Bose D., Dhawan H., Kandpal V., Vijay P. y Gopinatha M. (2018). Sustainable power generation from sewage and energy recovery from wastewater with variable resistance using microbial fuel cell. Enzyme. Microb. Tech. 118, 92-101. https://doi.org/10.1016/j. enzmictec.2018.07.007

Cárdenas J.F., Martínez V. y Acosta. (2011). Remoción de cromo (VI) por una cepa de Paecilomyces sp. resistente a cromato. Inf. Tecnol. 22 (4), 43-50. https://doi. org/10.4067/S0718-07642011000400006

Carmalin S. y Saikant S. (2016). Reduction of chromium (VI) with energy recovery using microbial fuel cell technology. Journal of Water Process Engineering 11,39-45. https://doi.org/10.1016/j.jwpe.2016.03.006

Chen H., Zheng P., Zhang J., Xie Z., Ji J.y Ghulam A. (2014). Substrates and pathway of electricity generation in a nitrification-based microbial fuel cell. Bioresource Technol. 161, 208-214. https://doi. org/10.1016/j.biortech.2014.02.081

Cheng S. y Logan B. (2011). Increasing power generation for scaling up single-chamber air cathode microbial fuel cells. Bioresource Technol. 102 (6), 4468-4473. https://doi.org/10.1016/j.biortech.2010.12.104

Cheung K. H. y Ji Dong Gu. (2007). Mechanism of hexavalent chromium detoxification by microorganisms and bioremediation aplication potential: A review. Int. Biodeter. Biodegr. 59 (1), 8-15. https:// doi,org/10.1016/j.ibiod.2006.05.002

Debabov V. G. (2008). Electricity from microorganisms. Microbiology 77 (2), 149-57. https://doi.org/10.1134/ S002626170802001X

Dhal B., Thatoi H., Das N. y Pandey B. (2013). Chemical and microbial remediation of hexavalent chromium from contaminated soil and mining / metallurgical solid waste: a review. J. Hazard Mater 250-251, 272-91. https://doi.org/10.1016/j.jhazmat.2013.01.048

Echeverry A. (2016). Caracterización de la cepa nativa Comomonas sp ARD-A3 y evaluación del potencial biorremediador de cromo. Tesis de Licenciatura. Facultad de Ciencias Naturales Biología, Universidad del Valle, Cali, Colombia, 30 pp.

Evelyne R. y Ravisanka V. (2014). Bioremediation of chromium contamination: a review. International Journal of Research in Earth and Environmental Sciences 1 (6), 20-26.

Guan C., Tseng Y., Tsang D., Hu A. y Yu C. (2019). Wetland plant microbial fuel cells for remediation of hexavalent chromium contaminated soils and electricity production. J Hazard Mater 365, 137-145. https:// doi.org/10.1016/j.jhazmat.2018.10.086

Guerrero D., Pinta J., Fernández P., Ibarguen E., Hidalgo S. y Burbano E. (2017). Eficiencia en la reducción de cromo por una bacteria silvestre en un tratamiento tipo batch utilizando como sustrato agua residual del municipio de pasto, Colombia. Universidad y Salud 19 (1), 102-15. https://doi.org/10.22267/ rus. 171901.74

He Z., Minteer S. y Angenent L. (2005). Electricity generation from artificial wastewater using an upflow microbial fuel cell. Environ. Sci. Technol. 39 (14), 5262-67. https://doi.org/10.1021/es0502876

Huang L., Chai X., Chen G. y Logan B. (2011). Effect of set potential on hexavalent chromium reduction and electricity generation from biocathode microbial fuel cells. Environ. Sci. Technol. 45 (11), 5025-31. https:// doi.org/10.1021/es103875d

Kanmani P., Aravind J. y Preston D. (2012). Remediation of chromium contaminants using bacteria. Int. J. Environ. Sci. Tech. 9 (1), 183-93. https://doi.org/10.1007/ s13762-011-0013-7

Kim C., Lee C., Song Y., Heo J., Choi S., Lim D., Cho J., Park C., Jang M. y Kim J. (2017). Hexavalent chromium as a cathodic electron acceptor in a bipolar membrane microbial fuel cell with the simultaneous treatment of electroplating wastewater. Chem Eng J. 328, 703-7. https://doi.org/10.1016/j. cej.2017.07.077

MADS, ANLA (2015). Resolución 1070 del 28 de agosto de 2015 "Por la cual se otorga un Permiso Marco de Recolección de Especímenes de Especies Silvestres de la Diversidad Biológica con Fines de Investigación Científica No Comercial y se toman otras determinaciones". Ministerio de Ambiente y Desarrollo Sostenible, Autoridad Nacional de Licencias Ambientales. 28 de agosto de 2015.

Li M., Zhou M, Tian X, Tan C., McDaniel K., Hassett D. y Gu T. (2018). Microbial fuel cell (MFC) power performance improvement through enhanced microbial electrogenicity.

Biotechnol Adv. 36 (4), 1316-27. https://doi.org/10.1016/j. biotechadv.2018.04.010

Li Y., Lu A., Ding H., Jin S., Yan Y., Wang C., Zen C. y Wang X. (2009). Cr(VI) reduction at rutile-catalyzed cathode in microbial fuel cells. Electrochem. Commun. 11 (7), 1496-99. https://doi.org/10.1016/j.elecom.2009.05.039

Liu Y., Song P., Gai R., Yan C., Jiao Y., Yin D., Cai L y Zhang L. (2019). Recovering platinum from wastewater by charring biofilm of microbial fuel cells (MFCs). J. Saudi Chem. Soc. 23 (3), 338-45. https:// doi.org/10.1016/j.jscs.2018.08.003

Loera A., Ramírez F., Avelar F. y Guerrero A. (2012). Biopelículas multi-especie: asociarse para sobrevivir. Investigación y Ciencia 54, 49-56.

Logan B., Hamelers B., Rozendal R., Schoder U., Keller J., Freguia S., Aelterman P., Verstraete W. y Rabaey K. 
(2006). Microbial fuel cells: methodology and technology. Environ. Sci. Technol. 40 (17). 5181-92. https:// doi.org/10.1021/es0605016

Loloei M., Rezaee A., Sabour A. y Aliofkhazraei M. (2017). Conductive microbial cellulose as a novel biocathode for $\mathrm{Cr}(\mathrm{VI})$ bioreduction. Carbohyd. Polym. 162, 56-61. https://doi.org/10.1016/j.carbpol.2017.01.046

Martínez-Santacruz C., Herrera-López D., GutiérrezHernández R. y Bello-Mendoza R. (2016). Tratamiento de agua residual doméstica mediante un reactor RAFA y una celda microbiana de combustible. Rev. Int. Contam. Ambie. 32 (3), 267-79. https://doi.org/10.20937/ RICA.2016.32.03.02

Mendoza J.,Vega M., Silveti A., Arriola J. y Pérez G. (2015). Estudio de la estructura de las biopelículas de pseudomonas putida mediante microscopia de fuerza atómica ( $\mathrm{afm}$ ) en presencia de permetrina y cipermetrina. international multilingual. Journal of Contemporary Research 3 (2), 120-30. https://doi. org/10.15640/imjcr.v3n2a12

Min B. y Logan B. (2004). Continuous electricity generation from domestic wastewater and organic substrates in a flat plate microbial fuel cell. Environ. Sci. Technol. 38 (21), 5809-14. https://doi.org/10.1021/es0491026

Mora A. y Bravo E. (2017). Aislamiento de microorganismos electrogénicos con potencial para reducir cromo hexavalente. Acta Biológica Colombiana 22 (1), 27-36. https://doi.org/10.15446/abc.v22n1.57189

Morais P., Branco R. y Francisco R. (2011). Chromium resistance strategies and toxicity: what makes Ochrobactrum tritici 5 bvl1 a strain highly resistant. BioMetals 24 (3), 401-10. https://doi.org/10.1007/s10534-011-9446-1

Okeke B. (2008). Bioremoval of hexavalent chromium from water by a salt tolerant bacterium Exiguobacterium sp. GS1. J. Ind Microbiol. Biot. 35 (12), 15711579. https://doi.org/10.1007/s10295-008-0399-5

Olarte S. y Cristiano J. (2012). Aplicación de microorganismos asociados a biopelículas. Revista Logos Ciencia y Tecnología 4 (1), 153-63. https://doi.org/10.22335/ rlct.v4i1.178

Oves M., Saghir M. y Zaidi A. (2013). Biosorption of heavy metals by Bacillus thuringiensis strain OSM29 originating from industrial effluent contaminated North Indian soil. Saudi J. Biol. Sci. 20 (2), 121-29. https:// doi.org/10.1016/j.sjbs.2012.11.006

Pant D., Gilbert V., Ludo D. y Karolien V. (2010). A review of the substrates used in microbial fuel cells (MFCS) for sustainable energy production. Bioresource Technol 101 (6), 1533-43. https://doi.org/10.1016/j. biortech.2009.10.017

Parkash A. (2016). Microbial fuel cells: a source of bioenergy. J. Microb. Biochem. Techno. 8 (3), 247-55. https://doi.org/10.4172/1948-5948.1000293
Ramírez A. y Benítez N. (2013). Tolerancia y reducción de cromo (VI) por Bacillus cereus B1, aislado de aguas residuales de una curtiembre. Revista de Ciencias 17 (2), 51-63.

Penteado E.D., Fernández-Marchante C.M., Zaiat M., González E.R. y Rodrigo M.A. (2016). On the effects of Ferricyanide as cathodic mediator on the performance of microbial fuel cells. Electocatalysis 8 (1), 59-66. https:// doi.org/10.1007/s12678-016-0334-x

Rangdale R. E., Richards R.H, y Alderman D. (1997). Minimum inhibitory concentrations of selected antimicrobial compounds against flavobacterium psychrophilum the causal agent of rainbow trout fry syndrome (RTFS). Aquaculture 158 (3-4), 193-201. https://doi. org/10.1016/S0044-8486(97)00202-0

Recillas S., Colón J., Casais E., Gonzáles E., Puntes V., Sánchez A. y Fuentes X. (2010). Chromium VI adsorption on cerium oxide nanoparticles and morphology changes during the process. J Hazard Mater 184 (1-3), 425-431. https://doi.org/10.1016/j. jhazmat.2010.08.052

Rehman A., Zahoor A., Muneer B. y Hasnain S. (2008). Chromium tolerance and reduction potential of a Bacillus sp.ev3 isolated from metal contaminated wastewater. B Environ. Contam. Tox. 81 (1), 25-29. https://doi.org/10.1007/s00128-008-9442-5

Reimers C., Tender L., Fertig S. y Wang (2001). Harvesting energy from the marine sediment-water interface. Environ. Sci. Technol. 35 (1), 192-95. https://doi. org $/ 10.1021 / \mathrm{es} 001223 \mathrm{~s}$

Revelo D.M. y Hurtado N. (2013). Celdas de combustible microbianas (CCMS): un reto para la remoción de materia orgánica y la generación de energía. Inf. Tecnol. 24 (6), 17-28. https://doi.org/10.4067/S071807642013000600004

Revelo D.M., Hurtado N., Ruiz J., Pabón L. y Ordoñez L. (2018). Bacterial diversity in the $\mathrm{Cr}(\mathrm{VI})$ reducing biocathode of a microbial fuel cell with salt bridge. Rev. Argent. Microbiol. 50 (3), 3-9. https://doi. org/10.1016/j.ram.2018.04.005

Sarangi A. y Krishnan C. (2008). Comparison of in Vitro $\mathrm{Cr}$ (VI ) reduction by CFEs of chromate resistant bacteria isolated from chromate contaminated soil. Bioresource Technol. 99 (10), 4130-37. https://doi. org/10.1016 / j.biortech.2007.08.059

Singh R., Dong H., Liu D., Zhao L., Marts A., Farquhar E,.Tierney D., Almquis C. y Briggs B. (2015). Reduction of hexabalente chromiun by the thermophilic methanogen Methanothermobacter termautotrophicus. Geochim. Cosmochi. Acta 148, 442-456. https://doi. org/10.1016/j.gca.2014.10012

Sophia C. y Sai S. (2016a). Modified microbial fuel cell for $\mathrm{Cr}(\mathrm{VI})$ reduction and simultaneous bio-electricity 
production. J. Envir. Che. Engin. 4, 2402-9. https:// doi.org/10.1016/j.jece.2016.04.025

Sophia C. y Sai S. (2016b). Reduction of chromium (VI) with energy recovery using microbial fuel cell technology. Journal of Water Process Engineering 11, 39-45. https://doi.org/10.1016/j.jwpe.2016.03.006

Suh M., Wikoff D., Lipworth L., Goodman M., Fitch S., Mittal L., Ring C. y Proctor D. (2019). Hexavalent chromium and stomach cancer: a systematic review and meta-analysis. Crit. Rev. Toxicol. 49 (2), 140-159. https://doi.org/10.1080/10408444.2019.1578730

Thatoi H., Das S., Mishra J., Prasad B. y Das N. (2014). Bacterial chromate reductase, a potential enzyme for bioremediation of hexavalent chromium: A review. J. Environ. Manage. 146, 383-99. https://doi. org/10.1016/j.jenvman.2014.07.014

Thygesen A., Finn W., Irini A., Booki M. y Anne B. (2011). Electricity generation by microbial fuel cells fuelled with wheat straw hydrolysate. Biomass Bioenerg. 35 (11), 4732-39. https://doi.org/10.1016/j. biombioe.2011.09.026

Tommasi T. y Lombardelli G. (2017). Energy sustainability of microbial fuel cell (MFC): A case study. J. Power Sources 356, 438-447. https://doi.org/10.1016/j. jpowsour.2017.03.122

Vejarano F., Benítez-Campo N., Bravo-Montaño E., Loaiza O. y Lizcano-Valbuena W. (2019). Electrochemical monitoring and microbial characterization of a domestic wastewater-fed microbial fuel cell inoculated with anaerobic sludge. Revista de Ciencias 22 (2), 13-32. https://doi.org/10.25100/ rc.v22i2.7910

Viti C., Marchi E., Decorosi F. y Giovannetti L. (2014). Molecular mechanisms of $\mathrm{Cr}(\mathrm{VI})$ resistance in bacteria and fungi. FEMS Microbiol. Rev. 38 (VI), 633-59. https://doi.org/10.1111 / 1574-6976.12051
Wang G., Huang H. y Zhang Y. (2008). Cathodic reduction of hexavalent chromium [Cr(VI)] coupled with electricity generation in microbial fuel cells. Biotechnol. Lett. 30 (11), 1959-66. https://doi.org/10.1007/ s10529-008-9792-4

Watson V. y Logan B. (2011). Analysis of polarization methods for elimination of power overshoot in microbial fuel cells. Electrochem. Commun. 13 (1), 54-56. https://doi.org/10.1016/j.elecom.2010.11.011

Wei V., Elektorowicz M. y Oleszkiewicz J. A. (2011). Influence of electric current on bacterial viability in wastewater treatment. Water Res. 45 (16), 5058-62. https://doi.org/10.1016/j.watres.2011.07.011

Wu X., Zhu X., Tianshun C., Zhang L., Jia H. y Wei P. (2015). Effect of acclimatization on hexavalent chromium reduction in a biocathode microbial fuel cell. Bioresource Technol. 180, 185-91. https://doi. org/10.1016/j.biortech.2014.12.105

Xing D., Zuo Y., Cheng S., Regan J. y Logan B. (2008). Electricity generation by Rhodopseudomonas palustris DX-1. Environ. Sci. Technol. 42, 4146-51. https://doi. org/10.1021/es800312v

Zhang L., Lu Z., Li D., Ma J., Song P., Huang G., Liu Y. y Cai L. (2016) Chemically activated graphite enhanced oxygen reduction and power output in catalyst-free microbial fuel cells. J Clean Prod. 115, 332-36. https:// doi.org/10.1016/j.jclepro.2015.12.067

Zumbair M. y Malik A. (2008). Chromate resistence, transport and bioreduction by Exiguobacterium sp. ZM-2 isolated form agricultural soil irrigated with tannery effluent. J. Basic Microb 48, 416-420. https:// doi.org/10.1002 / jobm.200800046 